\title{
Estudio de una selección de morteros de época romana de la provincia de Sevilla
}

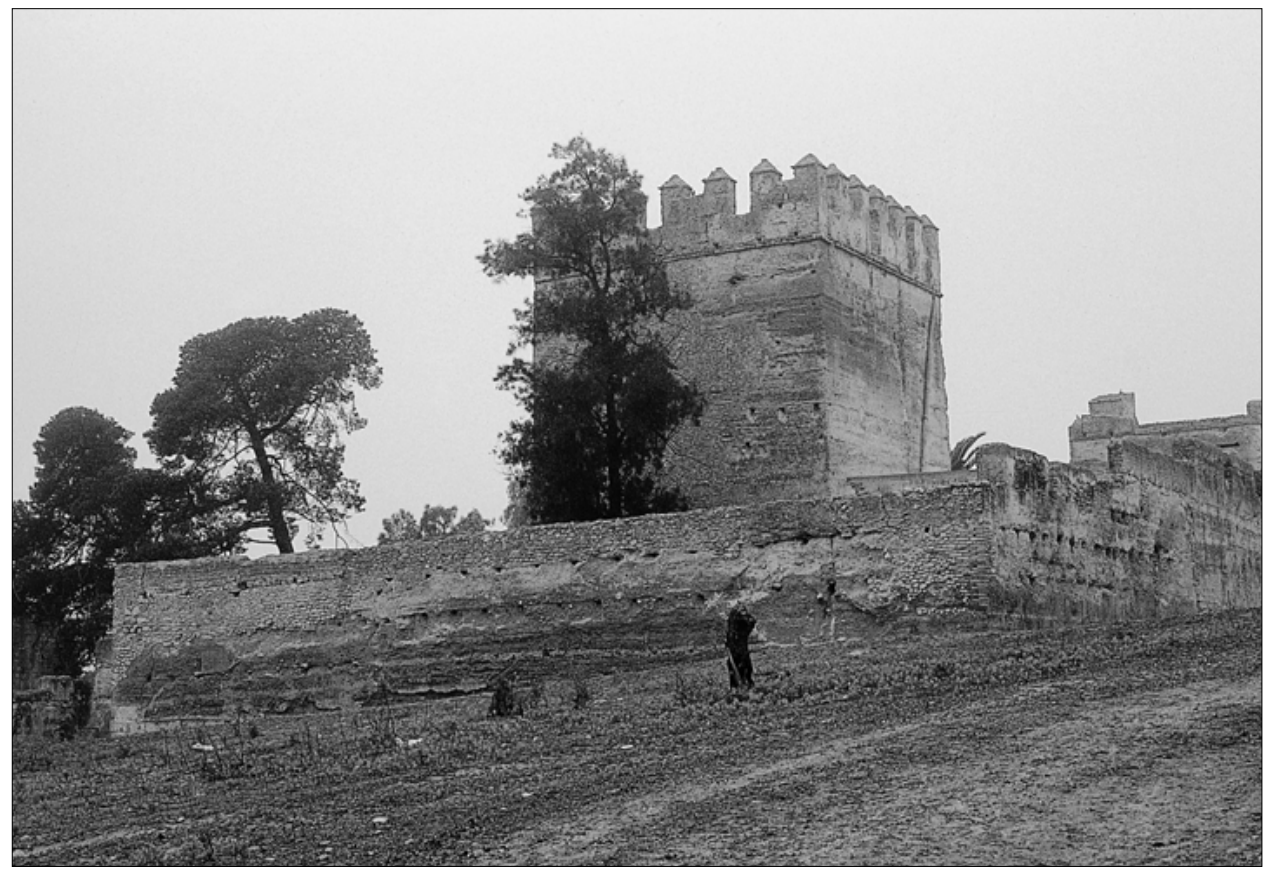

Flores Alés, V.

Guiraúm Pérez, A.

Dpto. Química Analítica

Barrios Sevilla, J. Dpto. Construcciones Arquitectónicas I Universidad de Sevilla

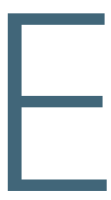

objeto de este trabajo es el estudio y caracterización de una serie de morteros de época romana procedentes de diversos yacimientos de la provincia de Sevilla. Se han estudiado las composiciones química y mineralógica, granulometrías y dosificaciones cal/agregado, siguiéndose la normativa actual de control de calidad. En la discusión de los resultados se tuvieron en cuenta las distintas zonas de procedencia y naturaleza geológica de las mismas.

\section{OBJETIVO}

En este trabajo se presentan los resultados del estudio de un conjunto de trece muestras de morteros de cal procedentes de diversos asentamientos romanos de la provincia de Sevilla, los yacimientos de procedencia 
son Itálica, en el municipio de Santiponce; Carmo, actual Carmona; Munigua, en el término de Villanueva del Río y Minas; Astigi, actual ciudad de Écija; y Arva, en la actual Alcolea del Río (FIGURA I).

Las muestras han sido designadas con la letra M seguida de una letra correspondiente a su procedencia y un número. En total se han estudiado trece muestras: cuatro de Itálica MII, MI2, MI3 y MI4, todas ellas procedentes de restos adheridos a piezas cerámicas; dos de Carmona, $\mathrm{MCl}$ y $\mathrm{MC2}$, la primera de ellas es un resto de estuco; una de Munigua, MMI, recogida directamente de una fábrica; dos muestras procedentes de Astigi MEI y ME2, ambas adheridas a ladrillos, procedentes de excavaciones arqueológicas en las calles Cava n³1 y Santa Cruz n 4 ; y cuatro muestras de Arva que también se encontraban adheridas a ladriIlos, fueron signadas como MAI, MA2, MA3 y MA4 respectivamente.

\section{INTRODUCCIÓN}

Para la caracterización de los materiales empleados en una construcción, es necesario un estudio pormenorizado y específico de cada uno de ellos. Dicho estudio conlleva, por tanto, el análisis de los morteros o aglomerantes utilizados en las fábricas; estos, una vez puestos en obra, se pueden considerar como un material propiamente dicho.

El estudio de los morteros antiguos es una cuestión que suscita el interés de los investigadores, especialmente desde el punto de vista de realizar aportaciones a la restauración y reposición de elementos deteriorados por el paso del tiempo.

El ligante más comúnmente empleado hasta el siglo XIX fue la cal, obtenida a partir del apagado de cal viva. En la investigación sobre morteros, uno de los puntos de mayor interés es el conocimiento de la cal empleada, aérea o hidráulica, dato que se puede conocer en función de la fracción de sílice soluble y del análisis térmico diferencial $(1,2)$.

Otros materiales utilizados como ligantes fueron el betún y el yeso, mientras que en la actualidad el ligante más extendido es el cemento. Curiosamente, los morteros de cemento sufren un deterioro con el tiempo mucho más acentuado que los de cal. Es más, los morteros de cal sufren una considerable mejora con el paso del tiempo. Así, morteros elaborados hace más de veinte siglos han llegado a nuestros días en un magnífico estado de conservación.

Actualmente, en los criterios sobre restauración de monumentos se plantean dos tendencias opuestas, en lo que respecta al tipo de mortero a emplear en las zonas donde éste se ha perdido. Por un lado se postula el empleo única y exclusivamente de morteros de cal, que reproduzcan exactamente la tecnología seguida en la construcción original; y de otro, hay restauradores que prefieren la combinación de morteros de
Figura 1. Mapa de la provincia de Sevilla, indicándose las distintas comarcas y lugares de procedencia de las muestras.

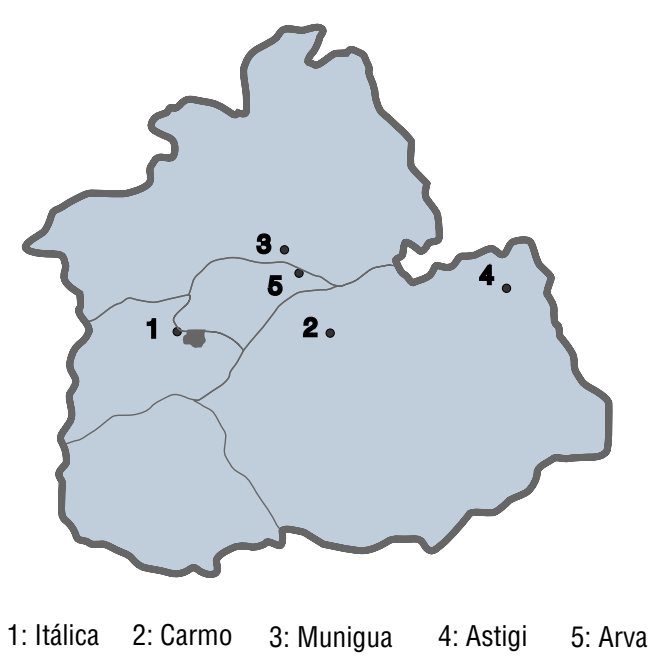

1: Itálica $\quad$ 2: Carmo $\quad$ 3: Munigua $\quad 4:$ Astigi $\quad$ 5: Arva

\begin{tabular}{|c|c|c|}
\hline \multicolumn{3}{|c|}{ Resultados de granulometría y curva granulométrica } \\
\hline Tamiz Une & $\% \mathrm{RP}$ & $\%$ PASA \\
\hline 5,00 & 2,2 & 97,8 \\
\hline 2,50 & 3,4 & 94,4 \\
\hline 1,25 & 6,6 & 87,8 \\
\hline 0,63 & 13,7 & 74,1 \\
\hline 0,32 & 33,2 & 40,9 \\
\hline 0,16 & 30,1 & 10,8 \\
\hline 0,08 & 10,8 & 0,0 \\
\hline
\end{tabular}

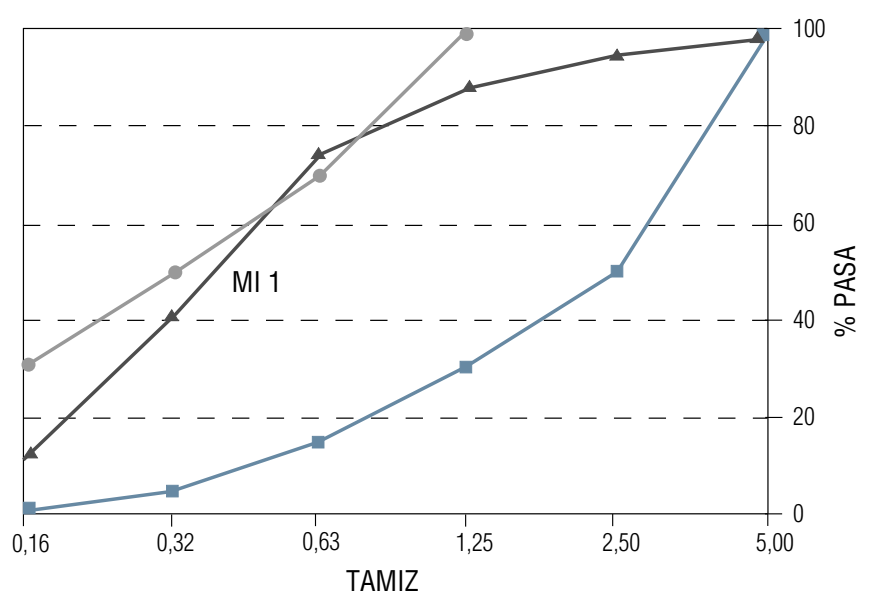

cemento en el interior de las fábricas y de morteros de cal en las fachadas. Los problemas planteados en restauración por los cementos tipo Portland son, principalmente, la excesiva tenacidad, el elevado coeficiente de expansión térmica, la incorporación de sales, con el consiguiente riesgo de lixiviación por solubilización parcial o total de componentes minerales, lo que provoca un incremento de porosidad y la consecuente 
$<$

는

ב
Figura 3. MORTERO CARMONA MC 1

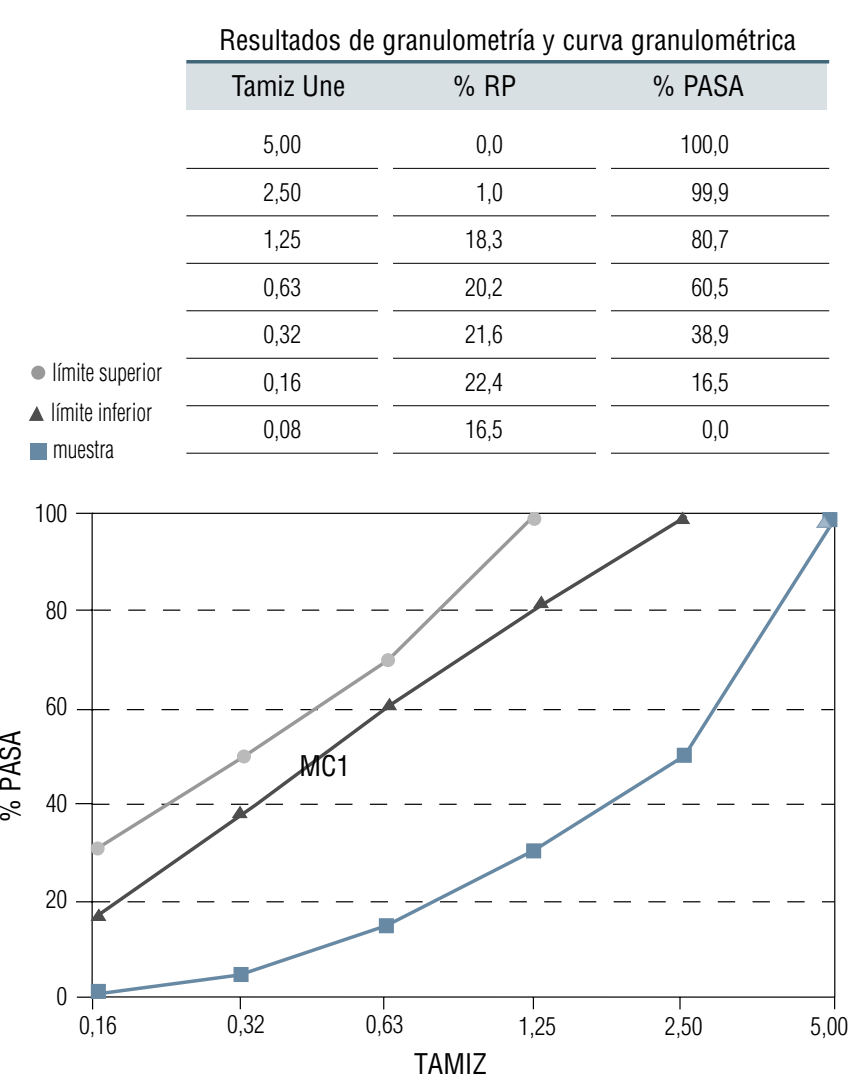

Figura 4. MORTERO MUNIGUA MM 1

\begin{tabular}{|c|c|c|c|}
\hline & \multicolumn{3}{|c|}{ Resultados de granulometría y curva granulométrica } \\
\hline & Tamiz Une & $\% \mathrm{RP}$ & $\%$ PASA \\
\hline & 5,00 & 2,1 & 97,9 \\
\hline & 2,50 & 10,0 & 87,9 \\
\hline & 1,25 & 27,0 & 60,9 \\
\hline & 0,63 & 27,1 & 33,8 \\
\hline \multirow{3}{*}{ - límite superior } & 0,32 & 19,0 & 14,8 \\
\hline & 0,16 & 8,6 & 6,2 \\
\hline & 0,08 & 6,2 & 0,0 \\
\hline
\end{tabular}

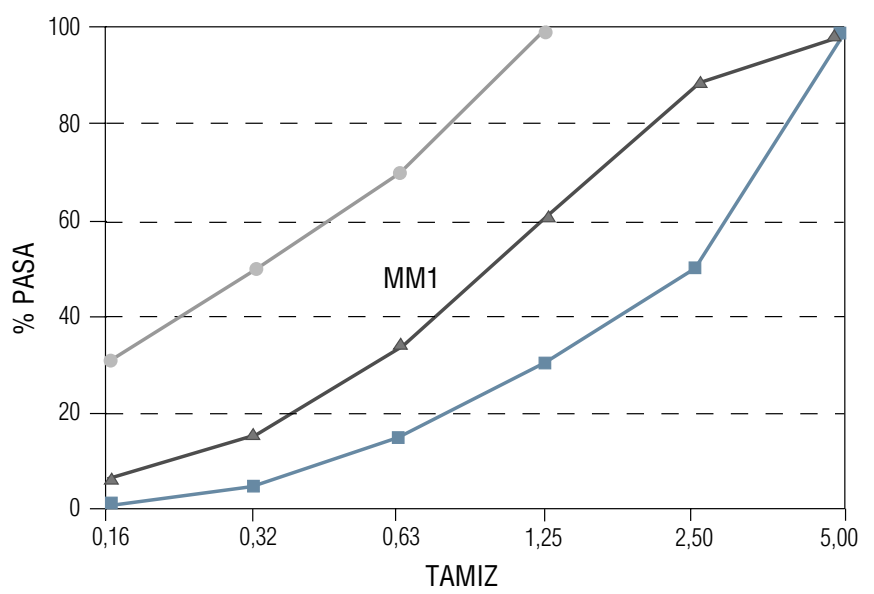

pérdida de resistencia; y por último la baja porosidad, que impide la eliminación de la humedad $(3,4)$.

\section{CARACTERÍSTICAS FÍSICAS Y MECÁNICAS}

Los morteros de cal se caracterizan por no tener una elevada resistencia mecánica y por el elevado tamaño de sus poros, lo que los hace sumamente permeables, lo cual, en determinadas circunstancias, puede facilitar la alteración de los mismos por agentes atmosféricos, en especial los ciclos de hielo-deshielo, y biológicos; por ello, la conservación de éstos depende, de forma muy directa, de la climatología. Por contra, la elevada permeabilidad y la baja resistencia mecánica facilitan, respectivamente, la fácil eliminación de humedad y producen una cierta capacidad de deformación, lo que contribuye a la durabilidad del material. En general los factores que pueden contribuir al deterioro se pueden agrupar en: $1^{\circ}$ Factores externos, $2^{\circ}$ Factores inherentes al mortero o motivados por restauraciones anteriores y $3^{\circ}$ Factores antropogénicos (5).

La dosificación de los morteros es muy variable, puesto que los secretos profesionales han tenido vigencia desde siempre. La determinación de la relación en peso de cal y agregado es fácil de obtener, siendo las proporciones aproximadas más habituales en morteros antiguos 1:1, 1:2 y 1:3 (cal/arena).

La cantidad de agua empleada en la dosificación, con toda probabilidad, era función de la experiencia, se considera que podía oscilar en torno al cincuenta por ciento en peso. Igualmente tiene una gran importancia en el comportamiento del mortero la granulometría de la arena empleada, cuya composición puede ir desde arenas muy finas hasta gravas con guijarros de gran tamaño utilizadas en las cimentaciones (6). En todo caso la dosificación y granulometría utilizadas han variado a lo largo de las distintas épocas de la historia, según las tecnologías imperantes y los lugares de aplicación.

\section{METOdología ANALÍTICA}

Los ensayos y análisis realizados sobre los distintos morteros han estado en función de la cantidad de muestra de que se dispuso. Al proceder algunas muestras de los restos adheridos a las piezas, no siempre ha resultado posible obtener cantidad suficiente para las determinaciones granulométricas

Se han determinado las relaciones cal/agregado de cada mortero, así como las composiciones química y mineralógica. Las curvas granulométricas se han comparado con las curvas límites admitidas por la normativa de calidad.

La relación cal/agregado se determinó por gravimetría tras ataque de la muestra con ácido clorhídrico (I:I). 
Los análisis químicos se realizaron por gravimetría para la sílice y pérdida al fuego (P.F.), espectroscopía de absorción/emisión atómica para determinación de aluminio, hierro, calcio, magnesio, manganeso, titanio, sodio y potasio y turbidimetría para determinación de sulfatos. La composición mineralógica se analizó por difracción de rayos $X$ mediante el método de polvo.

\section{DISCUSIÓN DE RESULTADOS DEL ANÁLISIS DE MORTEROS}

Antes de iniciar la discusión de resultados es importante señalar que el mortero MEI es significativamente distinto al resto, puesto que el árido está constituido por restos cerámicos, ello se debe a que procede de una conducción de agua, para las que se empleaba este tipo de agregado por sus mejores propiedades hidráulicas. En los demás casos el agregado es de naturaleza silícea, aportando de esta manera unas buenas resistencias mecánicas, así como establidad química al mortero.

En cinco de las muestras se dispuso de cantidad suficiente para realizar las curvas granulométricas, que se compararon con las granulometrías de las arenas empleadas en morteros según las condiciones descritas en la Tabla 3.2 de la NBE FL-90 (TABLA I)

En la TABLA 2 se presentan los resultados de las granulometrías obtenidas para las distintas muestras, quedando reflejados los \% de muestra que pasa por cada tamiz de la seríe correspondiente. Igualmente en las FIGURAS 2-6 se presentan las gráficas correspondientes a las curvas granulométricas representadas frente a los límites indicados por la NBE-FL 90.

A partir de los datos aportados por los estudios granulométricos, se puede indicar que tan sólo la muestra $\mathrm{MCl}$ tiene un tamaño máximo de árido de $5 \mathrm{~mm}$, tal y como se corresponde con las arenas, la muestra MEI es la que presenta una fracción mayor, alcanzando un tamaño máximo de $20 \mathrm{~mm}$. Así, la muestra $\mathrm{MCl}$ es la única que se adapta a las indicaciones de granulometrías para morteros. Por lo demás, las muestras MII y MEI son las que manifiestan algún otro incumplimiento, en ambos casos en el tamiz 0,63, en un caso el porcentaje de paso es superior al $70 \%$, mientras que en otro es inferior al 15\%.

\section{Figura 5. MORTERO ASTIGI ME 1}

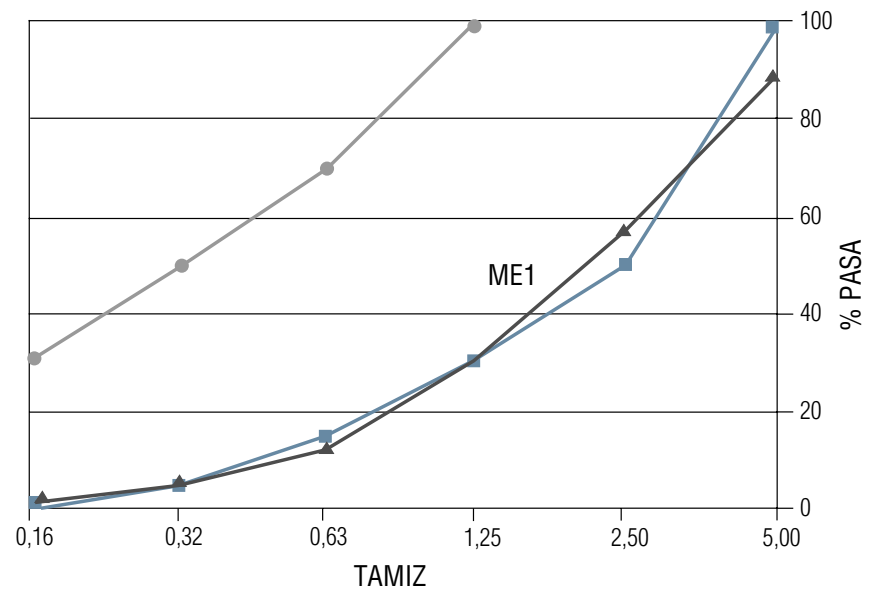

趐

\begin{tabular}{cccc}
\hline Tabla 1 & & \\
\hline $\begin{array}{c}\text { Tamiz } \\
\text { UNE } \mathbf{7 0 5 0}(\mathbf{m m})\end{array}$ & $\begin{array}{c}\text { \% que pasa } \\
\text { por el tamiz }\end{array}$ & \multicolumn{1}{c}{ Condiciones } \\
\hline 5,00 & $\mathrm{a}$ & $\mathrm{a}=100$ & \\
\hline 2,50 & $\mathrm{~b}$ & $60 \leq \mathrm{b} \leq 100$ & \\
\hline 1,25 & $\mathrm{c}$ & $30 \leq \mathrm{c} \leq 100$ & $\mathrm{c}-\mathrm{d} \leq 50$ \\
\hline 0,63 & $\mathrm{~d}$ & $15 \leq \mathrm{d} \leq 70$ & $\mathrm{~d}-\mathrm{e} \leq 50$ \\
\hline 0,32 & $\mathrm{e}$ & $5 \leq e \leq 50$ & $\mathrm{c}-\mathrm{e} \leq 70$ \\
\hline 0,16 & $\mathrm{f}$ & $0 \leq \mathrm{f} \leq 30$ & \\
\hline
\end{tabular}

Tabla 2. RESULTADOS DE LAS GRANULOMETRÍAS PARA CADA MUESTRA

\begin{tabular}{|c|c|c|c|c|c|}
\hline TAMIZ UNE & MI1 (\%pasa) & MC1 (\%pasa) & MM1 (\%pasa) & ME1 (\%pasa) & MA1 (\%pasa) \\
\hline 5,00 & 97,8 & 100,0 & 97,9 & 89,1 & 93,9 \\
\hline 2,50 & 94,4 & 99,9 & 87,9 & 56,5 & 82,3 \\
\hline 1,25 & 87,8 & 80,7 & 60,9 & 30,5 & 49,1 \\
\hline 0,63 & 74,1 & 60,5 & 33,8 & 11,9 & 26,2 \\
\hline 0,32 & 40,9 & 38,9 & 14,8 & 5,1 & 14,8 \\
\hline 0,16 & 10,8 & 16,5 & 6,2 & 1,3 & 5,1 \\
\hline 0,08 & 0,0 & 0,0 & 0,0 & 0,0 & 0,0 \\
\hline
\end{tabular}


$<$

ш

ב

\section{Figura 6. MORTERO ARVA MA 1}

Resultados de granulometría y curva granulométrica

\begin{tabular}{|c|c|c|}
\hline Tamiz Une & $\% R P$ & $\%$ PASA \\
\hline 5,00 & 6,1 & 93,9 \\
\hline 2,50 & 11,6 & 82,3 \\
\hline 1,25 & 33,2 & 49,1 \\
\hline 0,63 & 22,9 & 26,2 \\
\hline 0,32 & 11,4 & 14,8 \\
\hline 0,16 & 9,7 & 5,1 \\
\hline 0,08 & 5,1 & 0,0 \\
\hline
\end{tabular}

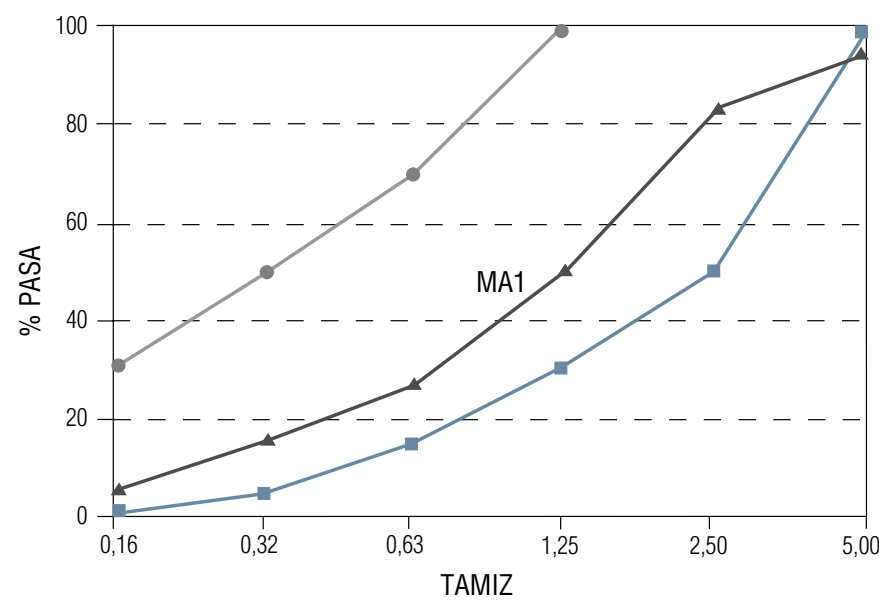

Salvo las indicaciones realizadas, las muestras cumplen las especificaciones para arenas, no sólo en las granulometrías, sino también en las relaciones entre porcentajes de paso.

Las relaciones cal-agregado determinadas no son totalmente exactas debido a que la dosificación de los morteros tampoco lo era (TABLA 3). En todo caso, como se puede comprobar, las relaciones son muy similares, $1: 1$ y $1: 2$.

La composición mineralógica de los morteros presenta unos porcentajes considerables de cuarzo y de calcita, esta última en mayor cantidad. Los feldespatos están presentes en muy pequeña cuantía, siendo destacables por superar el I0\% las muestras MMI y

Tabla 3. RELACIONES CAL/AGREGADOS DE LOS MORTEROS ESTUDIADOS

\begin{tabular}{|c|c|c|c|}
\hline MUESTRA & RELACIÓN & MUESTRA & RELACIÓN \\
\hline Ml1 & $1: 1$ & ME1 & $1: 2$ \\
\hline MI2 & 1:1 & ME2 & $1: 2$ \\
\hline $\mathrm{M} 13$ & $1: 1$ & MA1 & 1:1 \\
\hline MI4 & 1:1 & MA2 & $1: 2$ \\
\hline MC1 & $1: 2$ & MA3 & $1: 2$ \\
\hline MC2 & 1:2 & MA4 & 1:2 \\
\hline MM1 & $1: 2$ & & \\
\hline
\end{tabular}

MAI, las zonas de procedencia de estas dos muestra se encuentran muy cercanas entre sí; especialmente en Munigua, la litología se caracteriza por su naturaleza granítica, lo que justifica la presencia de feldespatos.

Como componentes minoritarios se encuentran hematite, en la muestra MEI, y dolomita, en ME2, MA3 y MA4.

La presencia de calcita en mayor medida que el cuarzo se explica por la naturaleza variada del agregado empleado, que aún en el caso de ser silíceo no es únicamente cuarzo. Los contenidos, tanto de calcita como de cuarzo, no presentan importantes divergencias, únicamente son destacables el bajo contenido en cuarzo de la muestra MEI (22\%) y los elevados de calcita de $\mathrm{MCl}, \mathrm{MC2}$ y $\mathrm{MEI}$ (73\%, 69\% y $73 \%)$.

El análisis general de los valores ofrece los siguientes datos:

\begin{tabular}{ll}
\hline & CUARZO \\
\hline Mínimo: & 22 \\
\hline Máximo: & 58 \\
\hline Media: & 38,1 \\
\hline Desv.std.: & 8,7 \\
\hline & \\
\hline & \\
\hline & CALCITA \\
\hline Mínimo: & 32 \\
\hline Máximo: & 73 \\
\hline Media: & 56,8 \\
\hline Desv.std.: & 10,5 \\
\hline
\end{tabular}

Al igual que el análisis mineralógico, el análisis químico no manifiesta importantes diferencias entre los valores de los diferentes componentes (TABLA 4).

El compuesto mayoritario es la sílice, siendo el valor significativamente más alto el de la muestra MMI $(67,04 \%)$, que es también la que tiene un contenido más alto de cuarzo.

Los óxidos metálicos (aluminio, hierro y titanio), de forma lógica, presentan valores bastante bajos, estando todos ellos en un rango similar; siendo destacables respecto del resto, los contenidos especialmente bajos en aluminio de la muestra MC2 (2,20\%) y en titanio de MMI (0,10\%).

Entre los contenidos de óxido cálcico destacan los de $\mathrm{MCl}$ y $\mathrm{MC2}$, que son los más elevados $(27,84 \%$ y 27,46\%), y el de MMI, que es el más bajo (12,00\%). En general, existe una relación homogénea entre los contenidos en este óxido y calcita. Los valores de P.F. son también muy similares y coherentes con los de óxido cálcico, guardando relación directa tanto los valores más elevados como los más bajos. Es importante señalar que, al ser los valores muy similares, las relaciones entre los distintos parámetros se manifiestan como tendencias. 


\begin{tabular}{|c|c|c|c|c|c|c|c|c|c|c|c|c|}
\hline & $\mathrm{SiO}_{2}$ & $\mathrm{Al}_{2} \mathrm{O}_{3}$ & $\mathrm{Fe}_{2} \mathrm{O}_{3}$ & $\mathrm{CaO}$ & $\mathrm{MgO}$ & MnO & PF & $\mathrm{TiO}_{2}$ & $\mathrm{Na}_{2} \mathrm{O}$ & $\mathrm{K}_{2} \mathrm{O}$ & $\mathrm{SO}_{3}$ & TOTAL \\
\hline Ml1 & 50,77 & 6,54 & 2,46 & 16,26 & 1,21 & 0,02 & 20,54 & 0,60 & 1,15 & 0,32 & 0,03 & 99,90 \\
\hline MI2 & 50,81 & 5,67 & 2,00 & 17,23 & 1,20 & 0,01 & 20,70 & 0,54 & 1,12 & 0,29 & 0,06 & 99,63 \\
\hline MI3 & 45,78 & 8,94 & 2,07 & 17,29 & 0,89 & 0,02 & 20,30 & 0,50 & 1,88 & 1,95 & 0,09 & 99,71 \\
\hline MI4 & 45,52 & 8,41 & 1,17 & 17,30 & 0,81 & 0,05 & 21,10 & 0,59 & 1,96 & 2,22 & 0,07 & 99,20 \\
\hline MC1 & 36,41 & 5,26 & 2,46 & 27,84 & 0,94 & 0,03 & 24,86 & 0,30 & 0,24 & 1,20 & 0,38 & 99,92 \\
\hline MC2 & 43,18 & 2,20 & 1,87 & 27,46 & 1,48 & - & 22,56 & 0,32 & 0,21 & 0,33 & 0,35 & 99,96 \\
\hline MM1 & 67,04 & 3,35 & 1,50 & 12,00 & 2,14 & 0,03 & 12,01 & 0,10 & 0,22 & 0,79 & 0,54 & 99,72 \\
\hline ME1 & 47,46 & 6,45 & 2,52 & 19,92 & 1,25 & 0,04 & 20,08 & 0,29 & 0,28 & 1,12 & 0,21 & 99,62 \\
\hline ME2 & 48,28 & 4,10 & 3,89 & 20,66 & 1,44 & 0,03 & 19,78 & 0,26 & 0,21 & 0,94 & 0,10 & 99,69 \\
\hline MA1 & 52,03 & 7,91 & 3,92 & 15,51 & 1,69 & 0,03 & 17,41 & 0,30 & 0,33 & 0,44 & 0,16 & 99,73 \\
\hline MA2 & 48,89 & 5,41 & 3,99 & 16,04 & 0,74 & 0,07 & 21,21 & 0,23 & 0,86 & 1,73 & 0,51 & 99,68 \\
\hline MA3 & 44,73 & 4,37 & 3,43 & 24,06 & 0,74 & 0,05 & 21,58 & 0,31 & - & 0,45 & 0,11 & 99,83 \\
\hline MA4 & 48,54 & 6,20 & 2,98 & 19,87 & 0,74 & 0,03 & 20,32 & 0,24 & 0,01 & 0,43 & 0,15 & 99,51 \\
\hline
\end{tabular}

\section{CONCLUSIONES}

El estudio de estos morteros ha revelado la similitud de las relaciones cal/agregado empleadas en los distintos asentamientos, así como el uso de agregados silíceos, con bajo o nulo contenido en sustancias agresivas, siendo reseñable los bajos contenidos en yeso y óxidos metálicos.

Estas circunstancias hacen patente una buena tecnología constructiva, aún más si se comparan los resultados con otros estudios realizados sobre morteros de la zona, en los que se puede comprobar que se mantiene la homogeneidad en las dosificaciones para áridos de tamaño máximo similar, así lo demuestran los estudios de Puertas y colaboradores sobre los morteros de Itálica, los cuales presentan relaciones cal/agregado de 1:1 y 1:3 para tamaño máximo superior a $4 \mathrm{~mm}(4)$.

Dosificaciones clásicas de morteros de cal y arena I:I y $1: 2$, como las obtenidas, son asimilables según algunos autores, desde un punto de vista conservador, a morteros de los tipos M- 10 y M- I 5 (7). De esta forma se puede realizar suposiciones "a priori" sobre la resistencia mecánica de los morteros sin recurrir a ensayos destructivos que demanden una mayor cantidad de muestra.

El empleo de arenas con granulometrías muy próximas a las descritas actualmente como óptimas por la normativa para dichos usos, continuas y homogéneas, pone de manifiesto nuevamente la calidad de las técnicas constructivas.

Desde el punto de vista del análisis mineralógico, el bajo o nulo contenido en feldespatos se puede considerar también como un indicador de la calidad de los agregados, puesto que su presencia en mayores cantidades produciría una caída de las resistencias mecánicas.

Todo ello permite constatar una cuidadosa selección en los materiales empleados, correcta dosificación y homogeneidad, así como una buena puesta en obra. Todas estas características y otras como son la buena cocción y extinción de la cal, la selección de materiales para mezclas (terracotas, tejas,...) han sido estudiadas y constatadas por otros autores $(8,9,10)$

\section{Bibliografía}

I. FRIZOT M., L'analyse des mortiers antiques, ICCROM Symp., Roma 1981, pp. 331-339.

2. ERNESTO BORRELLI y col., Realización de muestras de mortero de cal aérea, III Cong. Rehab. Patrimonio, Granada 1996, pp. 307-3।3.

3. PERONI y col. Mortars, ciments and grouts used in conservation of historic buildings, 1981.

4. PUERTAS y col. Characterization of mortars from the mosaics of Itálica, III Int. Symp. on Conservation of Monuments, Venize 1994, pp. 577-583.

5. LUXÁN M.P. y col., Estudios preliminares sobre morteros de cal, III Cong. Rehab. Patrimonio, Granada 1996, pp. 320-324.
6. MARTíN A., Ensayos y experiencias de alteración, Fund. Areces, 1990, p. 533.

7. BARRIOS J., Apuntes de materiales de construcción: morteros y hormigones, Universidad de Sevilla, 1994.

8. MALINOUSKI R . y col., Durability of roman mortars and concretes, RILEM, Praga 1961.

9. FURLAN V., BISSEGER P., "Les mortiers anciens", Revue Suisse d'Art et d'Archéologie n³2, 1975, pp. 1-14.

10. ÁLVAREZ GALINDO J.l. y col., "Historia de los morteros", Bol. Inst. Andaluz de Patrimonio, n¹3, Dic. 1995, pp. 52-59. 


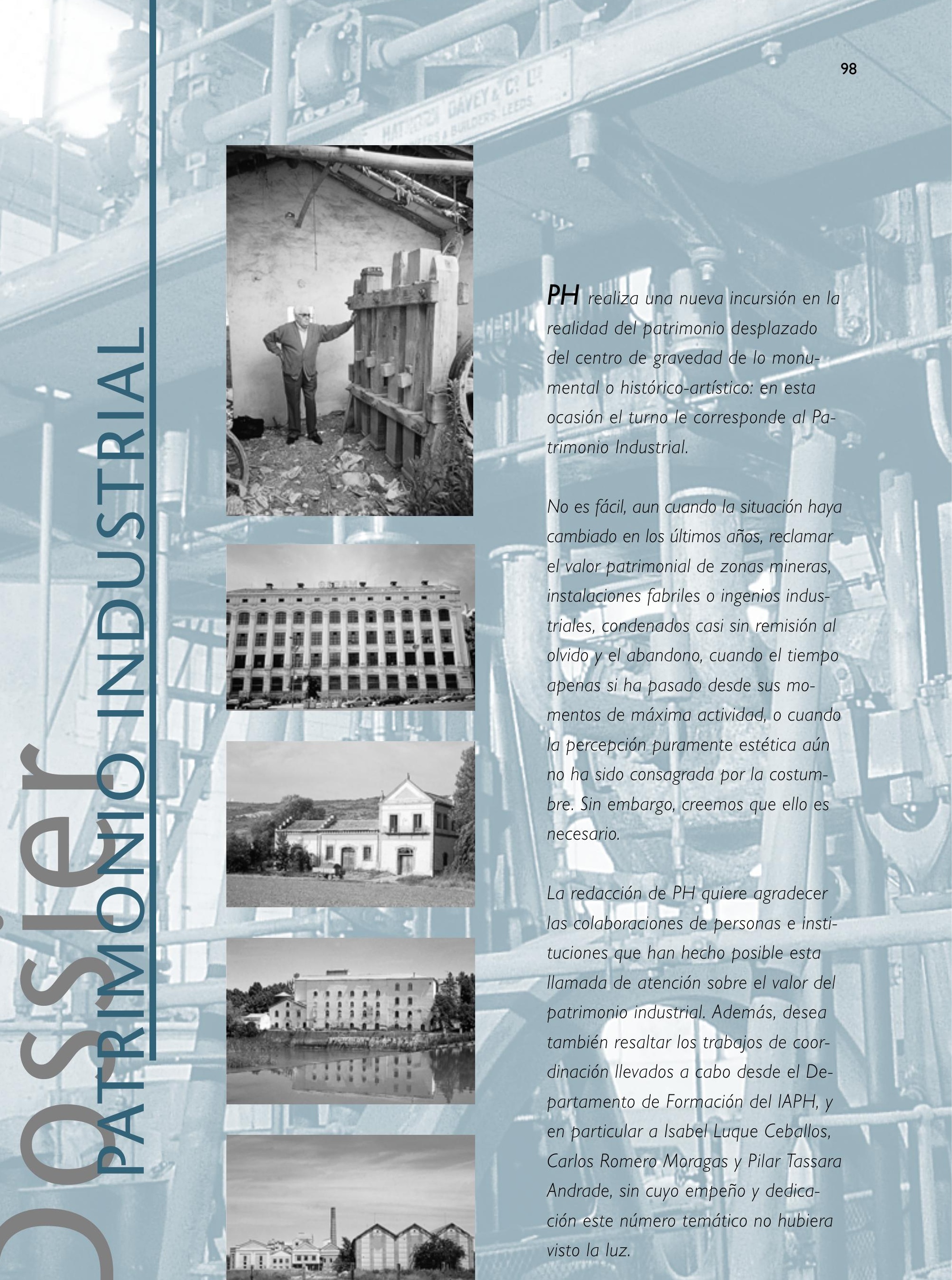

